\title{
Bridging charge-orbital ordering and Fermi surface instabilities in half-doped single-layered manganite $\mathrm{La}_{0.5} \mathrm{Sr}_{1.5} \mathrm{MnO}_{4}$
}

\author{
D. V. Evtushinsky, ${ }^{1}$ D. S. Inosov,${ }^{1}$ G. Urbanik,,${ }^{1,2}$ V. B. Zabolotnyy,${ }^{1}$ R. Schuster, ${ }^{1}$ P. Sass, ${ }^{1}$ T. Hänke, ${ }^{1}$ \\ C. Hess, ${ }^{1}$ B. Büchner, ${ }^{1}$ R. Follath, ${ }^{3}$ P. Reutler, ${ }^{4}$ A. Revcolevschi, ${ }^{4}$ A. A. Kordyuk, ${ }^{1,5}$ and S. V. Borisenko ${ }^{1}$ \\ ${ }^{1}$ Institute for Solid State Research, IFW Dresden, P. O. Box 270116, D-01171 Dresden, Germany \\ ${ }^{2}$ Institute of Experimental Physics, University of Wroclaw, pl. Maxa Borna 9, 50-204 Wroclaw, Poland \\ ${ }^{3}$ BESSY GmbH, Albert-Einstein-Strasse 15, 12489 Berlin, Germany \\ ${ }^{4}$ Laboratoire de Physico-Chimie de l'Etat Solide, Université Paris-Sud XI, 91405 Orsay Cédex, France \\ ${ }^{5}$ Institute of Metal Physics of National Academy of Sciences of Ukraine, 03142 Kyiv, Ukraine
}

PACS numbers:

Density waves are inherent to the phase diagrams of materials that exhibit unusual, and sometimes extraordinarily useful properties, such as superconductivity and colossal magnetoresistance [1, 2, 3, 4]. While the pure charge density waves (CDW) are well described by an itinerant approach [5, 6], where electrons are treated as waves propagating through the crystal, the charge-orbital ordering (COO) is usually explained by a local approach [7], where the electrons are treated as localized on the atomic sites. Here we show that in the half-doped manganite $\mathrm{La}_{0.5} \mathrm{Sr}_{1.5} \mathrm{MnO}_{4}$ (LSMO) the electronic susceptibility, calculated from the angle-resolved photoemission spectra (ARPES), exhibits a prominent nesting-driven peak at one quarter of the Brillouin zone diagonal, that is equal to the reciprocal lattice vector of the charge-orbital pattern. Our results demonstrate that the Fermi surface geometry determines the propensity of the system to form a COO state which, in turn, implies the applicability of the itinerant approach also to the COO.

In the present study we apply three different techniques, angle-resolved photoemission spectroscopy (ARPES), scanning tunneling microscopy (STM), and transport measurements, to exactly the same single crystals of $\mathrm{La}_{0.5} \mathrm{Sr}_{1.5} \mathrm{MnO}_{4}$ (LSMO), a renown representative of manganites, which exhibits a prominent transition to the charge-orbital ordered (COO) state [8, 9, 10, 11]. The STM results, summarized in Fig. 1, reveal high quality of the cleaved surface. Fig. 1(b) shows a typical STM topographic image obtained on an in situ cleaved surface of the manganite. The measured step heights are equal to $n \times(0.62 \pm 0.02) \mathrm{nm}, n \in \mathbb{Z}$, that is a multiple of half the c lattice constant [see Fig. 1(b), (c)]. This implies that the cleavage takes place between the adjacent La,Sr oxide layers [see Fig. 1(a)]. Note that in contrast to Ref. 12, our data reveal well resolved surfaces and steps, both above and below the COO temperature, $T_{\mathrm{COO}}=230 \mathrm{~K}$. The measured roughness of the terraces, observed in our data, was usually $0.1 \mathrm{~nm}$, indicating previous assessments $(0.6 \mathrm{~nm})[10,12]$ to be largely overestimated. A large defect-free portion of the surface is revealed by the atomically resolved STM image is shown in Fig. 1(d). The atomically resolved topography is highlighted by the Fourier transform of the STM image [Fig. 1(e)]. The absence of additional peaks in low-energy electron diffraction (LEED)
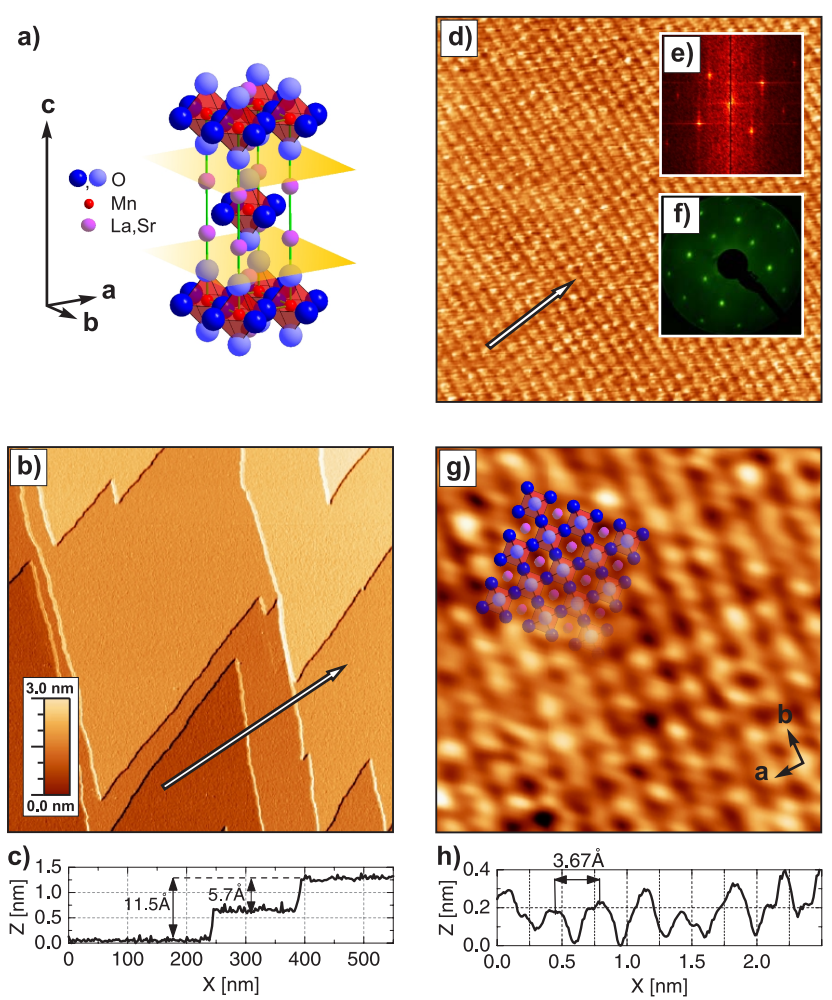

Fig. 1 (color online). Crystalline structure and surface quality of $\mathrm{La}_{0.5} \mathrm{Sr}_{1.5} \mathrm{MnO}_{4}$ single crystals. STM topographic images, taken at $300 \mathrm{~K}$. The sample is positively biased relative to the STM tip $(\mathrm{V}=0.5 \mathrm{~V}, \mathrm{I}=0.2 \mathrm{nA})$.(a) The crystalline structure of $\mathrm{La}_{0.5} \mathrm{Sr}_{1.5} \mathrm{MnO}_{4}$. The elementary cell is marked by green lines, and cleavage planes are shown in yellow. (b) $1 \mu \mathrm{m} \times 1 \mu \mathrm{m}$ image of atomically flat micrometer-sized terraces separated by steps. (c) The line profile measured on the figure (b), highlighting half-unitcell high steps. (d) $10 \mathrm{~nm} \times 10 \mathrm{~nm}$ atomic-resolution image measured on the flat terrace from figure (b). (e) The Fourier transform of the STM image (d). (f) LEED image, taken at $100 \mathrm{eV}$ with the same orientation as the STM image, indicates a reconstructionfree surface. $(\mathrm{g})$ Higher-magnification image of an area from the figure (d). (h) The line profile measured on the figure (d), showing the atomic modulations. The distance between the adjacent maxima corresponds to the value of the lattice constant.

image [Fig. 1(f)] confirms the absence of any significant surface reconstruction. Fig. $1(\mathrm{~g})$ shows a higher-magnified image with a superimposed cartoon of the crystalline structure. To the best of our knowledge, this is the first realspace observation of the La,Sr oxide layer in single-layered 

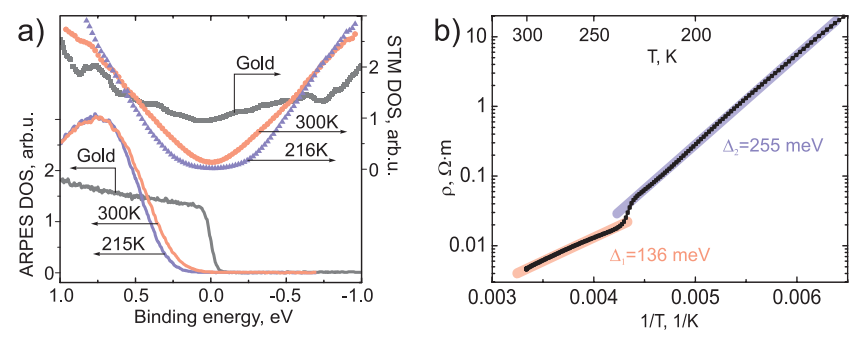

Fig. 2 (color online). The similarity of the surface and bulk electronic structures is indicated by the consistency among the conductive properties and behavior of the DOS near the Fermi level. (a) The DOS extracted from the tunneling (averaged over a whole momentum space) and photoemission ( $\Gamma$-pocket) data above and below $T_{\mathrm{COO}}$. Spectroscopies on gold are shown for calibration. (b) $\ln \rho$ plotted versus $1 / T$. Linear fit above and below $T_{\mathrm{COO}}$ is shown as underlying thick lines.

\section{manganites.}

In Fig. 2(a) we show both, photoemission and tunneling spectroscopic data taken from the surface discussed above. The extracted densities of states (DOS), shown on the same energy scale, exhibit similar behaviour near the Fermi level. This semiconducting behaviour is in agreement with the temperature dependence of the in-plane resistivity $\rho$, which is linear in $\ln \rho$ versus $1 / T$ coordinates [see Fig. 2(b)]. Remarkably, the values of the energy gap, determined from the resistivity measurements $\left(\Delta_{1}=136 \mathrm{meV}\right.$ above and $\Delta_{2}=255 \mathrm{meV}$ below $T_{\mathrm{COO}}$ respectively), are consistent with our spectroscopic data, indicating that in LSMO the surface is a good representative of the bulk. This is in agreement with the recent comparative X-ray scattering studies of bulk and surface of the same material [10].

Now we turn to the reciprocal space image of the LSMO electronic structure. In Fig. 3(a)-(e) we present ARPES intensity maps. Since the spectral weight on the Fermi level is nearly absent [Fig. 2(a)], these maps represent the momentum distribution of the intensity integrated in a finite energy window below the Fermi level (see caption to Fig. 3). In the following we will refer to these maps as remnant Fermi surface (FS) [13]. Since the energy gap, estimated from both spectroscopic and transport data, is rather small in comparison with the bandwidth, the remnant FS is very close to the hypothetical FS of the non-gapped parent metal [14] and thus has similar properties. As for each particular excitation energy the photoemission matrix elements highlight and suppress different parts of the spectrum [15, 16], which may lead to false conclusions as for the geometry of the electronic structure, we carried out the series of measurements at different photon energies and light polarizations, also analyzing data taken in different Brillouin zones [see Fig. 3(a)-(e)]. The compilation of the data allows us to conclude that the remnant FS of LSMO consists of a large hole-like barrel centered at the $\mathrm{K}$ point and a small electron-like pocked centered at the $\Gamma$ point [see Fig. 3(a)(e)], just as in bilayer manganites in their metallic state [17, 18]. We found that the remnant FS sheets are well described by the following tight binding formula:

$$
\begin{gathered}
\epsilon\left(k_{x}, k_{y}\right)=d_{0}+d_{1}\left(\cos k_{x} a+\cos k_{y} a\right)+d_{2} \cos k_{x} a \cos k_{y} a \\
+d_{3}\left(\cos 2 k_{x} a+\cos 2 k_{y} a\right)+d_{4}\left(\cos 2 k_{x} a \cos k_{y} a\right. \\
\left.\quad+\cos k_{x} a \cos 2 k_{y} a\right)+d_{5} \cos 2 k_{x} a \cos 2 k_{y} a
\end{gathered}
$$

with $d_{0}=3.050, d_{1}=-4.638, d_{2}=3.022, d_{3}=1.006$, $d_{4}=-1.720, d_{5}=0.660$ for the K-barrel, and $d_{0}=5.33$, $d_{1}=-2.974, d_{2}=0, d_{3}=0, d_{4}=0, d_{5}=0$ for the $\Gamma$-pocket, where $a=3.865 \AA[11]$ is a lattice constant [19, 20]. It is interesting, that the area enclosed by the contours (49\% of the Brillouin zone) is close to the halffilling and since this will not change much in the absence of the energy gap [14], this is in contrast to the nominal doping level of $1 / 2$ electron per $\mathrm{Mn}$ atom, which corresponds to $25 \%$ of the Brillouin zone. Here we also note, that at particular polarizations and excitation energies an apparent anisotropy of the photoemission signal shows up [see, e.g. Fig. 3.(c), (e)], which might point to the sample anisotropy in the a-b plane [21]. However, rotation of the sample by $90^{\circ}$ did not affect the measured signal, so we may conclude that the mentioned anisotropy of the spectra arises purely from the anisotropy of the photoemission matrix elements. The absence of any inherent sample anisotropy both above and below $T_{\mathrm{COO}}$ is in agreement with the presence of small domains with different orientations of the COO pattern [22], see Fig. 4(a) and (b).

With the LSMO electronic structure at hand we try to understand the origin of the charge-orbital ordering in this compound in the framework of an itinerant approach. Thereto we refer to the experience gained from studying compounds that exhibit another type of ordering - charge density wave (CDW). In these compounds, the major reason that drives the formation of the ordering is well understood: if the electronic susceptibility (Lindhard function) of the unreconstructed system possesses a strong peak at a particular wave vector, the system is likely to develop a density wave order at this vector [5, 6]. On the basis of the band dispersion extracted from ARPES data we have calculated the susceptibility of the hypothetical LSMO metal by a procedure similar to that described in Ref.23. The result of these calculations is shown in Fig. 3 (f), (g). The susceptibility turnes out to have a prominent nesting-driven peak very close to the vector

$$
\mathbf{q}=\frac{\mathbf{a}^{*}}{4}+\frac{\mathbf{b}^{*}}{4}
$$

where $\mathbf{a}^{*}=2 \pi \mathbf{a} /|\mathbf{a}|^{2}$ and $\mathbf{b}^{*}=2 \pi \mathbf{b} /|\mathbf{b}|^{2}$ are the lattice vectors in the reciprocal (momentum) space, while $\mathbf{a}$ and $\mathbf{b}$ are lattice vectors in the direct (coordinate) space [24]. Generally speaking, the FS nesting occurs when two replicas with opposite Fermi velocities meet each other. It means that the nesting is strong when FS sheets of the same origin (both hole-like or both electron-like) are externally tangent, or when the FS sheets of different origin (one hole-like and another electron-like) are internally tangent. In Fig. 4(c), FS (green solid line) and its replica shifted by the vector 
a)

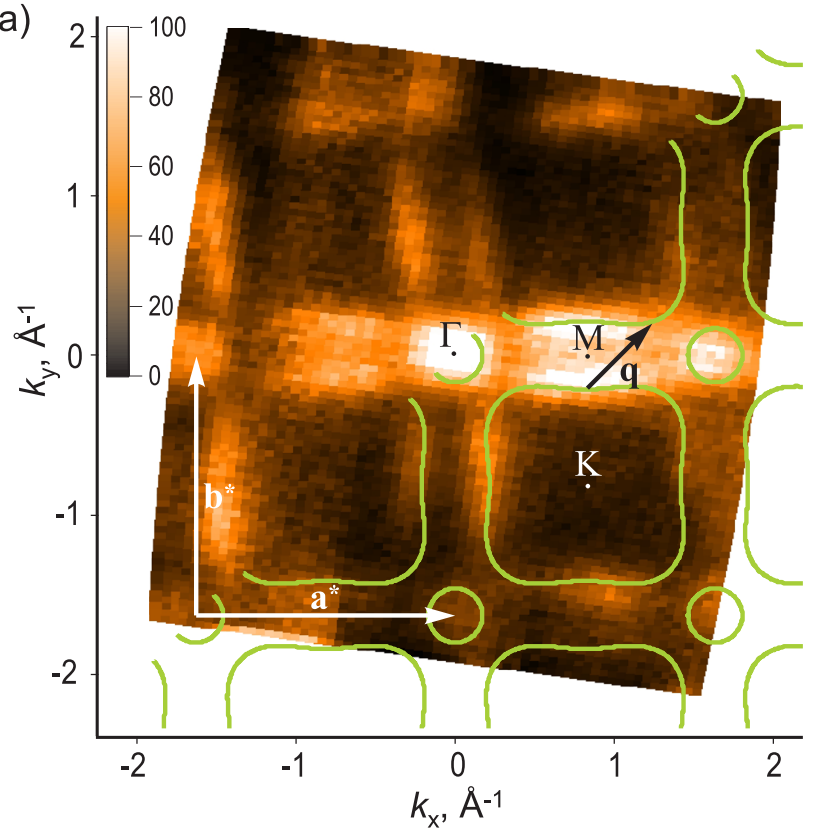

b)

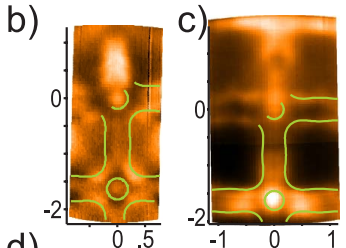

d)

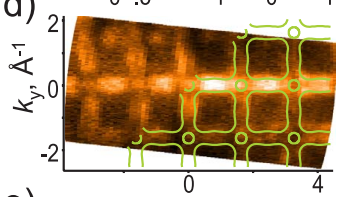

e)

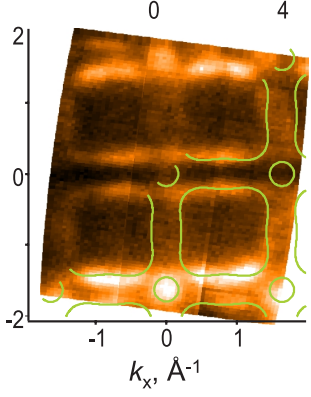

f)

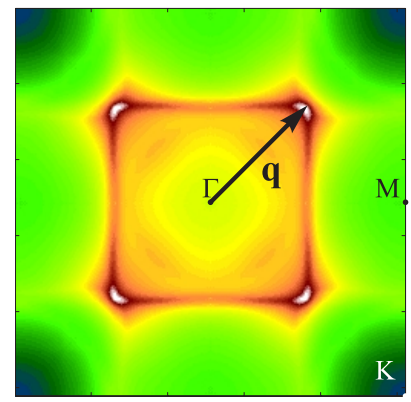

g)

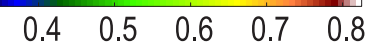

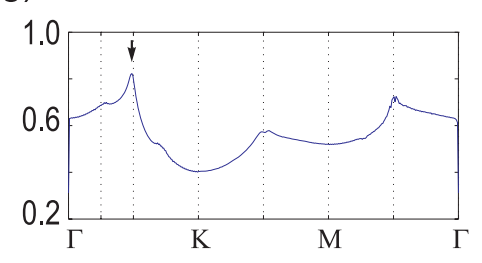

Fig. 3 (color online). Electronic structure and susceptibility of $\mathrm{La}_{0.5} \mathrm{Sr}_{1.5} \mathrm{MnO}_{4}$. Band dispersion is derived from ARPES spectra, taken at different experimental conditions, to neutralize effect of matrix elements. The electronic susceptibility, calculated on the basis of the revealed band dispersion, shows a prominent peak at the vector $\mathbf{q}=\mathbf{a}^{*} / 4+\mathbf{b}^{*} / 4$. The photoemission intensity, integrated in energy window of $70 \mathrm{meV}$ width, centered at $190 \mathrm{meV}$ below Fermi level, is shown in panels (a) - (e) for different excitation energies and light polarizations: (a) linear horizontal, $157 \mathrm{eV}, 300 \mathrm{~K}$; (b) linear horizontal, $37 \mathrm{eV}, 190 \mathrm{~K}$; (c) linear horizontal, $70 \mathrm{eV}, 195 \mathrm{~K}$; (d) linear horizontal, $200 \mathrm{eV}, 300 \mathrm{~K}$; (e) linear vertical, $157 \mathrm{eV}, 300 \mathrm{~K}$. Contours of the remnant Fermi surface, which consists of a small round electron-like pocket centered at the $\Gamma$ point and a large square hole-like barrel centered at the K point, are presented in panels (a) - (e) by green lines. The calculated susceptibility is shown in panel (f), and its profile along the high-symmetry directions is shown in ( $\mathrm{g}$ ).

q (orange thin line) are shown [panel (d) corresponds to a different orientation of the charge-orbital pattern]. We see that the FS of LSMO is nested with the vector $\mathbf{q}-$ there are two regions that contribute mostly to the peak in the susceptibility: the electron-like $\Gamma$-pocket fits inside the rounded corner of the square-shaped hole-like barrel, and the convex corner of the hole-like barrel fits inside its concave part.

The nesting vector we observe corresponds to the lattice vector of the charge-orbital superstructure, directed along zigzag chains [see Fig. 4(a)]

$$
\lambda=2 \mathbf{a}+2 \mathbf{b} .
$$

Let's prove that $\boldsymbol{\lambda}$ corresponds to the nesting vector $\mathbf{q}$. Eqs. (2) and (3) in conjunction with $\mathbf{a} \| \mathbf{a}^{*}$ and $\mathbf{b} \| \mathbf{b}^{*}$ result in $\mathbf{q} \| \boldsymbol{\lambda}$. Taking the modulus from Eqs. (2) and (3), and keeping in mind that in our case $|\mathbf{a}|=|\mathbf{b}|$ as well as $\left|\mathbf{a}^{*}\right|=\left|\mathbf{b}^{*}\right|$, we get $|\mathbf{q}|=(2 \sqrt{2})^{-1}\left|\mathbf{a}^{*}\right|$ and $|\lambda|=2 \sqrt{2}|\mathbf{a}|$. Finally recalling that $\mathbf{a}^{*} \cdot \mathbf{a}=2 \pi$, yields $\mathbf{q} \cdot \lambda=2 \pi$.

The found correspondence hints that the propensity of the system to form a $\mathrm{COO}$ state is determined by the FS instability due to the nesting-driven peak in the electronic susceptibility. Furthermore, significant changes in the physical properties, observed in LSMO upon charge-orbital ordering [8, 9], are similar to those observed upon charge ordering [1, 6, 25, 26] in classical CDW systems. Although $\mathrm{CDW}$ and $\mathrm{COO}$ are very similar, no relation between the
$\mathrm{COO}$ and the fermiology has been established to date to our knowledge, albeit some theoretical works have predicted a possibility of the description of the $\mathrm{COO}$ phenomena via the band approach [27] as an alternative to the existing local approach [7]. Our observations provide such a link by suggesting that FS instabilities due to either Peierls-like or other mechanisms can serve as a possible origin of the COO.

The project is part of the FOR538 and was supported by the DFG under Grants No. KN393/4 and BO1912/21. We thank R.Hübel for technical support, A. Narduzzo and R. Schneider for resistivity measurements. ARPES experiments were performed using the " $1^{3}$ ARPES" end station at the Berliner Elektronenspeicherring-Gesellschaft für Synchrotron Strahlung m.b.H. (BESSY).

[1] E. Morosan et. al., Superconductivity in $\mathrm{Cu}_{x} \mathrm{TiSe}_{2}$, Nature Phys. 2, 544 (2006)

[2] T. Hanaguri, C. Lupien, Y. Kohsaka, D.-H. Lee, M. Azuma, M. Takano, H. Takagi and J. C. Davis, A 'checkerboard' electronic crystal state in lightly hole-doped $\mathrm{Ca}_{2-x} \mathrm{Na}_{x} \mathrm{CuO}_{2} \mathrm{Cl}_{2}$, Nature 430, 1001 (2004)

[3] G. C. Milward, M. J. Calderon and P. B. Littlewood, Electronically soft phases in manganites, Nature 433, 607 (2005)

[4] Y. Tokura and N. Nagaosa, Orbital Physics in TransitionMetal Oxides, Science 288, 462 (2000) 
a)

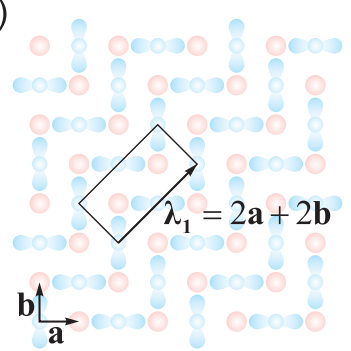

c)

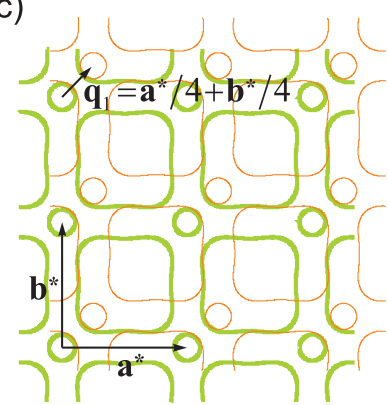

b)

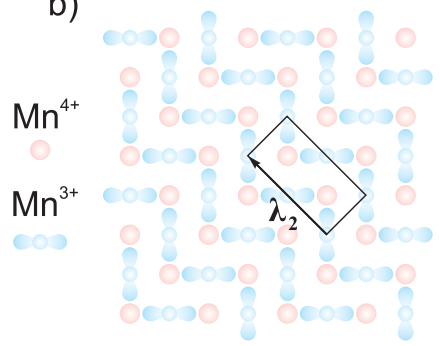

d)

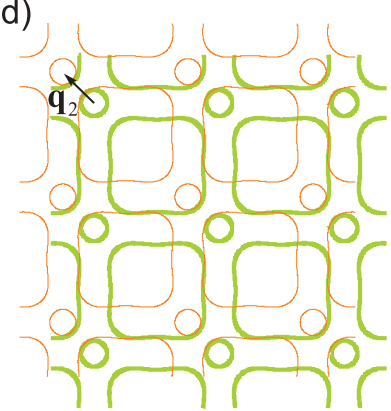

Fig. 4 (color online). The modulation in direct space and the corresponding nesting in reciprocal space; illustration of two possible orientations of the charge-orbital pattern. (a), (b) Realspace pattern of the charge-orbital order with lattice vectors $\lambda_{1,2}= \pm 2 \mathbf{a}+2 \mathbf{b}$ along zigzags. (c), (d) Fermi surface (green solid lines) and its replicas (thin orange lines) displaced by the vectors $\mathbf{q}_{1,2}= \pm \mathbf{a}^{*} / 4+\mathbf{b}^{*} / 4$. Note that the observed nesting vector, $\mathbf{q}_{1,2}$ corresponds to the periodicity of the charge-orbital pattern along zigzags, $\lambda_{1,2}:\left|\mathbf{q}_{1,2}\right|=(2 \sqrt{2})^{-1}\left|\mathbf{a}^{*}\right|,\left|\lambda_{1,2}\right|=2 \sqrt{2}|\mathbf{a}|$, and $\mathrm{q}_{1,2} \| \lambda_{1,2}$.

[5] S.-K. Chan and V. Heine, Spin density wave and soft phonon mode from nesting Fermi surfaces, J. Phys. F: Metal Phys. 3, 795 (1973);

J. A. Wilson, Charge-density waves in the $2 \mathrm{H}-\mathrm{TaSe} 2$ family: Action on the Fermi surface, Phys. Rev. B 15, 5748 (1977);

[6] S. V. Borisenko et. al., Pseudogap and Charge Density Waves in Two Dimensions, Phys. Rev. Lett. 100, 196402 (2008);

D. S. Inosov et. al., Fermi surface nesting in several transition metal dichalcogenides, arXiv:0805.4105. New J. Phys. in press (2008)

[7] J.van den Brink, G. Khaliullin, D. Khomskii, Charge and Orbital Order in Half-Doped Manganites, Phys. Rev. Lett. 83, 5118 (1999)

[8] Y. Moritomo et.al., Magnetic and electronic properties in hole-doped manganese oxides with layered structures: $\mathrm{La}_{1-x} \mathrm{Sr}_{1+x} \mathrm{MnO}_{4}$, Phys. Rev. B 51, 3297 (1995)

[9] D. Senff et.al., Spin-Wave Dispersion in Orbitally Ordered $\mathrm{La}_{1 / 2} \mathrm{Sr}_{3 / 2} \mathrm{MnO}_{4}$, Phys. Rev. Lett. 96, 257201 (2006);

D. Senff et.al., Melting of magnetic correlations in chargeorbital ordered $\mathrm{La}_{1 / 2} \mathrm{Sr}_{3 / 2} \mathrm{MnO}_{4}$ : Competition of ferromagnetic and antiferromagnetic states, Phys. Rev. B 77, 184413 (2008)

[10] Y. Wakabayashi et. al., Surface effects on the orbital order in the single-layered manganite $\mathrm{La}_{0.5} \mathrm{Sr}_{1.5} \mathrm{MnO}_{4}$, Nature Materials 6, 972 (2007)

[11] P. Reutler et. al., Growth of $\mathrm{La}_{1-x} \mathrm{Sr}_{1+x} \mathrm{MnO}_{4}$ single crystals

and characterization by scattering techniques, J. of Crystal Growth 249, 222 (2003)

[12] Plummer et. al., The next 25 years of surface physics, Prog. Surf. Science 67, 17 (2001)

[13] F. Ronning et.al., Photoemission Evidence for a Remnant Fermi Surface and a d-Wave-Like Dispersion in Insulating $\mathrm{Ca}_{2} \mathrm{CuO}_{2} \mathrm{Cl}_{2}$, Science 282, 2067 (1998)

[14] Taking into account small magnitude of the gap ( $\Delta \sim$ $150 \mathrm{meV})$ and large values of the Fermi velocity $\left(v_{\mathrm{F}} \sim\right.$ $4 \mathrm{eV} \cdot \AA$ ) in the case of LSMO, one may estimate the difference between the observed remnant FS and the hypothetical FS of the noninteracting metal as much smaller than $\Delta / v_{\mathrm{F}} \sim 0.04 \AA^{-1}$.

[15] S. V. Borisenko et. al., Estimation of matrix-element effects and determination of the Fermi surface in $\mathrm{Bi}_{2} \mathrm{Sr}_{2} \mathrm{CaCu}_{2} \mathrm{O}_{8+\delta}$ systems using angle-scanned photoemission spectroscopy, Phys. Rev. B 64, 094513 (2001)

[16] D. S. Inosov et. al., Momentum and Energy Dependence of the Anomalous High-Energy Dispersion in the Electronic Structure of High Temperature Superconductors, Phys. Rev. Lett. 99, 237002 (2007);

D. S. Inosov et. al., Excitation energy map of high-energy dispersion anomalies in cuprates, Phys. Rev. B 77, 212504 (2008)

[17] Y.-D. Chuang et.al., Fermi Surface Nesting and Nanoscale Fluctuating Charge/Orbital Ordering in Colossal Magnetoresistive Oxides, Science 292, 1509 (2001);

[18] N. Mannella et.al., Nodal quasiparticle in pseudogapped colossal magnetoresistive manganites, Nature 438, 474 (2005)

[19] Values of the tight-binding coefficients were adjusted to yield the best approximation of the band dispersion in the vicinity of the Fermi level, i.e. the input required to calculate the response of the system.

[20] We observe no significant temperature dependence of the intensity distribution in the remnant FS maps.

[21] There are reports on the in-plane sample anisotropy, bound to the formation of the single-domain $\mathrm{COO}$ pattern, in the similar compound - $\mathrm{Eu}_{0.5} \mathrm{Ca}_{1.5} \mathrm{MnO}_{4}$, see Y. S. Lee et. al., InPlane Anisotropy of the Electronic Structure for the Chargeand Orbital-Ordered State in Half-Doped Manganite with Layered Structure, Phys. Rev. Lett. 97, 077203 (2006)

[22] T. Ishikawa, K. Ookura and Y. Tokura, Optical response to orbital and charge ordering in a layered manganite: $\mathrm{La}_{1 / 2} \mathrm{Sr}_{3 / 2} \mathrm{MnO}_{4}$, Phys. Rev. B 59, 8367 (1999)

[23] D. S. Inosov et. al., Relation between the one-particle spectral function and dynamic spin susceptibility of superconducting $\mathrm{Bi}_{2} \mathrm{Sr}_{2} \mathrm{CaCu}_{2} \mathrm{O}_{8+\delta}$, Phys. Rev. B 75, 172505 (2007)

[24] Deviation of the revealed peak position from commensurate value $\mathbf{a}^{*} / 4+\mathbf{b}^{*} / 4$ is about $2 \%$, i.e. within error bars.

[25] D. V. Evtushinsky et. al., Pseudogap-Driven Sign Reversal of the Hall Effect, Phys. Rev. Lett. 100, 236402 (2008)

[26] M. Naito and S. Tanaka, Electrical Transport Properties in $2 \mathrm{H}-\mathrm{NbS}_{2},-\mathrm{NbSe}_{2},-\mathrm{TaS}_{2}$ and $-\mathrm{TaSe}_{2}$, J. Phys. Soc. Jpn. 51, 219 (1982)

[27] S. Yarlagadda, P. B. Littlewood, M. Mitra, R. K. Monu, Orbital ordering in undoped manganites via a generalized Peierls instability, arXiv:0808.1174 (2008);

D. V. Efremov and D. I. Khomskii, Orbital ordering in manganites in the band approach, Phys. Rev. B 72, 012402 (2005) 